\title{
Recycled Thermoplastic Composites from Fishing Rope with Crushed Rice Hull
}

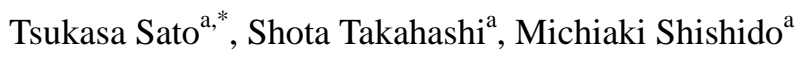 \\ ${ }^{a}$ National Institute of Technology, Tsuruoka College, 104 Sawada, Inooka, Tsuruoka, Yamagata 997-8511, Japan \\ *Corresponding Author: tsato@tsuruoka-nct.ac.jp
}

\begin{abstract}
In order to extend the utilization of recycled plastics made from fishing ropes wasted at sea side, composites with crushed rice hull (RH) were manufactured and mechanical behavior for the composites was analyzed. Particle size of crushed RH were $710 \sim 350 \mu \mathrm{m}$ (RH1), 180 160 $\mu \mathrm{m}$ (RH2), and about $17 \mu \mathrm{m}$ as an averaged diameter (RH3). Fourier transformed infrared spectrometry (FTIR) and differential scanning calorimetry (DSC) measurements revealed that the fishing ropes used in this study were composed of polypropylene (PP) and polyethylene (PE) materials. The tensile and bending tests were conducted based on the Japanese Industrial Standards of JIS-K7162 and 7171. Results of the tensile and bending tests means that the modulus of the composite increased by adding the RH. In general the composites became brittle by adding the RH. Effect of RH particle size on mechanical properties was more remarkable for tensile mode than bending. As particle size decreased the tensile and bending elastic modulus of the composites increased. While degree of elongation was decreased with decreasing of RH size. .
\end{abstract}

Keywords: polymer recycling, rice hull, composite, mechanical behavior.

\section{Introduction}

\subsection{Problem of sea waste by plastics}

The environmental destruction by the increase in plastic wastes has been a problem all over the world. Recently, the cases of sea waste are also increasing matter. Of these, the amount of the waste fishing rope is large and waste treatment of it is difficult. Moreover, since they have been placed under environment for a long time, mechanical properties of them may be degraded. Therefore, a technology for strength improvement is needed for those recycled products. Use of a filler is the most popular in such technology.

\subsection{Rice hull as plastics filler}

Investigations of technical potentiality for using rice hull (RH) particles as high-performance materials have been received much attention from the view points of utilization of agricultural wastes. The amount of it is estimated to be 2.6 million tons per year in Japan ${ }^{(1)}$. The rice hull contains about $20 \mathrm{wt} \%$ of inorganic compound and $80 \%$ of organic compound $^{(2)}$. Main of the inorganic compound is silica $\left(\mathrm{SiO}_{2}\right)$. Silica possess network structure by joining -Si-O- and forms crystalline, which give unique properties. Since silica possess their own advantages, the utilization of filler of silica in plastics should give the benefits. The RH fillers/polymer composites are expected to be high tensile and compression and elastic modulus ${ }^{(3-6)}$. The authors have chemically analyzed the waste ropes and revealed that these are composed of thermoplastic synthetic polymers ${ }^{(7)}$. Thermoplastic resins can be recycled by melting and processing at high temperature under high pressure. If the composites of $\mathrm{RH}$ and the resins obtained from fishing ropes would be high-performance materials, they might be contribute solving the environmental problem. Sato et al investigated basic characterizations of the composites from viewpoints of mechanical properties ${ }^{(7)}$. The main objective of this study is to investigate the breaking stress, degree of elongation and elastic modulus, in tensile and bending mode, for the RH/thermoplastic resin recycled from fishing ropes. In addition, comparison of such mechanical behavior by differing particle size of RH was also investigated. 


\section{Methods}

\section{$2.1 \quad$ Materials}

The $\mathrm{RH}$ was dried for 8 hours at $120^{\circ} \mathrm{C}$, and crushed by using the ball mill and then sieved. Three types of particle sizes were used, i. e., 710 350 $\mu \mathrm{m}$ (RH1), 180 $160 \mu \mathrm{m}$ (RH2), and about $17 \mu \mathrm{m}$ as an averaged diameter

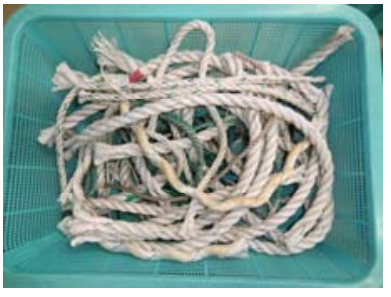

a

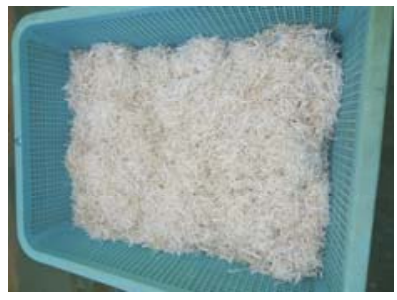

b

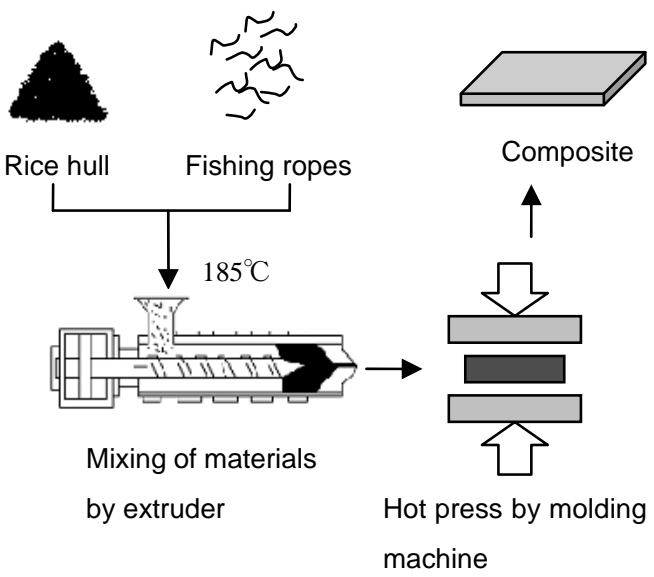

Fig. 2. Process of manufacturing the composites.

Fig. 1. Fishing ropes used in this study; before (a) and after (b) cutting.

(RH3). The recycled resin used was made from waste fishing ropes, which have been drifted ashore on the coats and have been collected by authors. They were cut into pieces by the shear and then washed by water several times followed by drying under an atmosphere. Figure 1 shows the photographs of fishing ropes before and after the cutting. Analysis of fourier transformed infrared spectrometry (FTIR) and differential scanning calorimetry (DSC) were conducted in order to obtain information about chemical components and thermal properties of the fishing ropes, respectively. Spectra of the FTIR were obtained between from 4000 to $750 \mathrm{~cm}^{-1}$ of wave numbers with a resolution of $4 \mathrm{~cm}^{-1}$ using attenuated total reflection method. DSC thermograms were obtained from 100 to $200^{\circ} \mathrm{C}$ at a heating rate of $10^{\circ} \mathrm{C} / \mathrm{min}$ under the nitrogen atmosphere.

\section{$2.2 \quad$ Processing}

The pieces of fishing roples were introduced at $185^{\circ} \mathrm{C}$ toward extrusion machine (Imoto Seisakusho PPKR IMC-1895) with certain content of $\mathrm{RH}$ particles. Moldings were conducted using pressure of $10 \mathrm{MPa}$ at the temperature from 175 to $180^{\circ} \mathrm{C}$ for $10 \mathrm{~min}$ (Fig. 2). The dimension of the test specimens was $80(w) \times 5(d) \times 0.5(t)$ $\mathrm{mm}$ for tensile and 40(w) x 10(d) x 1(t) mm for bending tests, respectively.

\subsection{Mechanical tests}

The tensile and bending tests were conducted based on the Japanese Industrial Standards of JIS-K7162 and 7171. These tests were operated by using Shimadzu EZ Test (type EZ-S) with $100 \mathrm{~N}$ maximum load. The crosshead speed was $1 \mathrm{~mm} / \mathrm{min}$ for tensile and $10 \mathrm{~mm} / \mathrm{min}$ for bending modes, respectively.

\section{Results and Discussion}

Figure 3 shows the typical infrared spectroscopic data for the fishing ropes obtained. The absorption bands at 2900, and $1450-1550 \mathrm{~cm}^{-1}$ correspond to vibration of chemical bond between carbon and hydrogen atoms, which suggesting hydrocarbon structure such as polyethylene(PE) and polypropyrene(PP). The band at 1370 implies existence of methyl group $\left(\mathrm{CH}_{3}\right)$, which is the part of structure of PP. Figure 4 shows the DSC curve for the fishing ropes. The endothermic peaks were observed at about 138 and $170^{\circ} \mathrm{C}$,

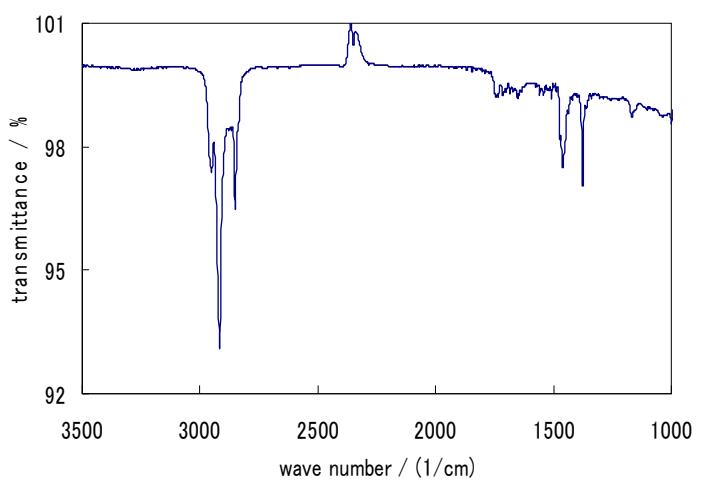

Fig. 3. FTIR spectrum for the fishing ropes used in this study. 


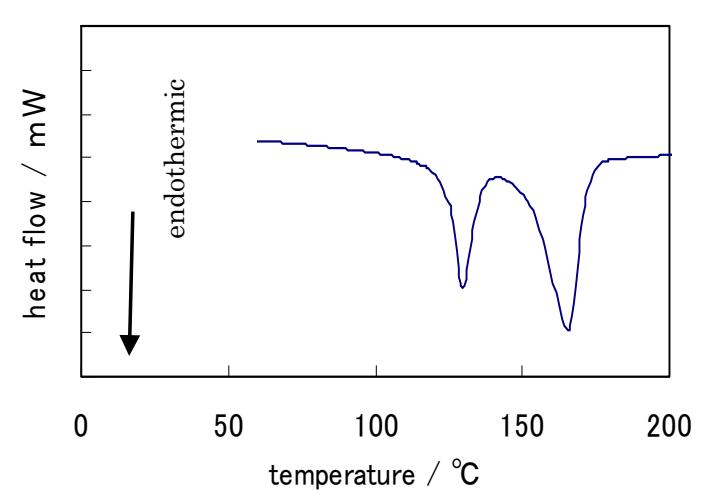

Fig. 4. DSC curve for the fishing ropes.

which correspond to melting peaks of the PE and PP, respectively. From the results of FTIR and DSC, it was found out that the fishing rope used in this study was composed of PP and PE materials.

Figure 5 shows the typical plots of stress-strain curves by the tensile mode using the testing machine for the composites with the RH1 of 50 phr content (phr: parts per hundred parts of resin). The tests were conducted using at least 5 pieces of the samples. It can be seen that stress increased markedly as the strain increases. As the values of stress passed through the maximum point on the curves, the specimen was broken and the stress suddenly decreased.

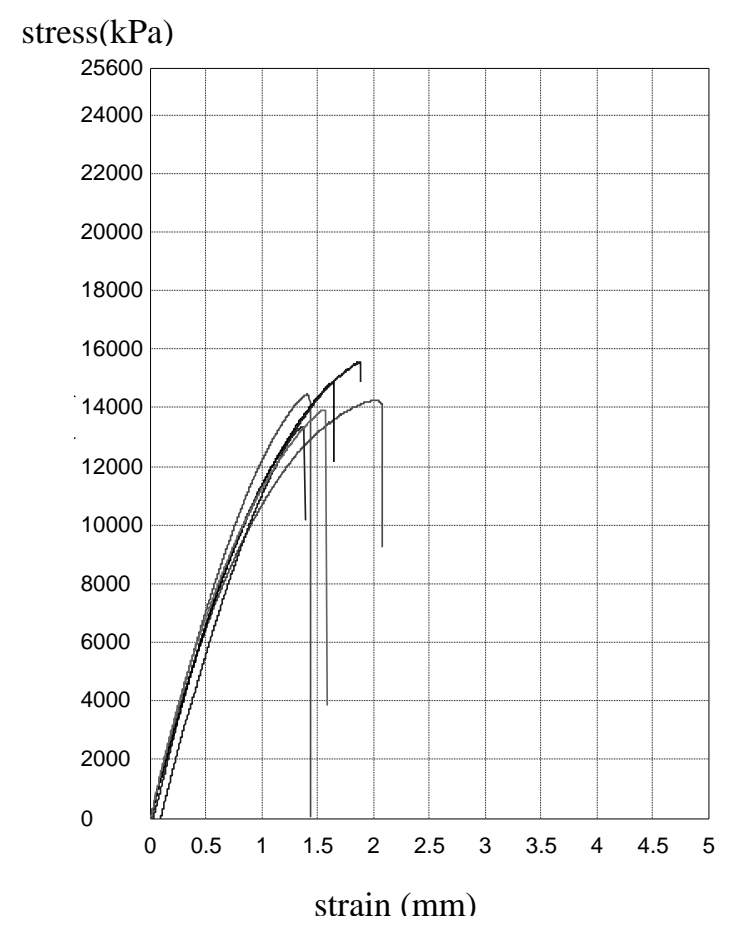

Fig. 5 Typical plots of stress-strain curves by the tensile mode for the composites with the RH1 of 50 phr.
Figure 6 (a)-(c) show results of the stress at maximum point(breaking point), degree of elongation at maximum point and elastic modulus for $50 \mathrm{phr}$ of various $\mathrm{RH}$ containing composites under the tensile mode, respectively. As shown in (a) of the figure, the stress was decreased by adding $\mathrm{RH}$ than the sample which consists only of plastics(no RH). Intensity of RH3 composite sample is higher than other two composite samples. The strain was remarkably decreased by adding $\mathrm{RH}$. Of these samples RH3 composite was remarkable (c).

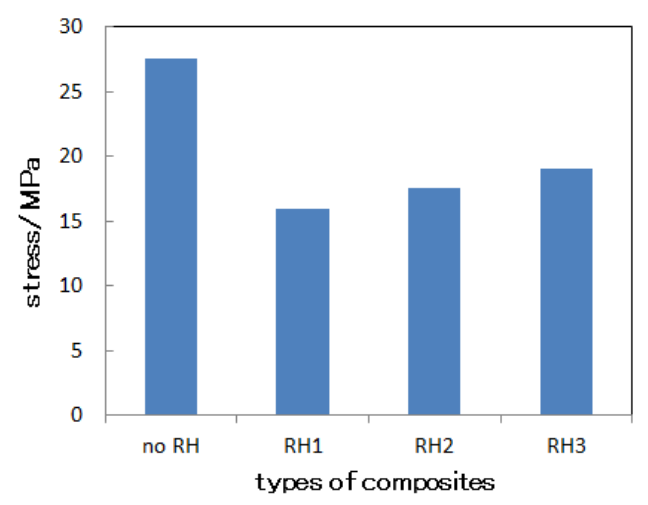

(a) Stress at maximum point

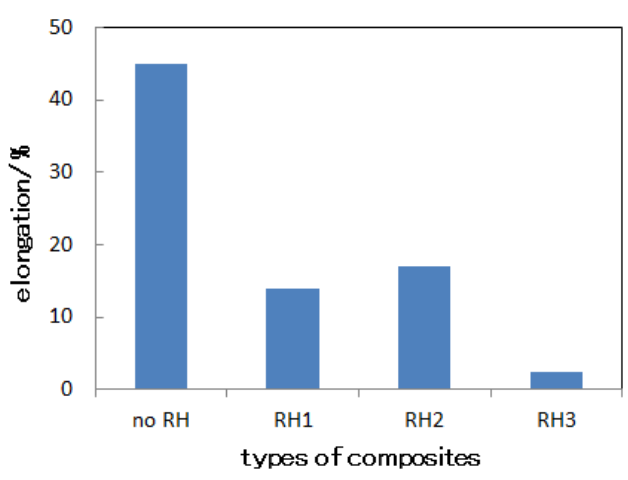

(b)elongation at maximum point

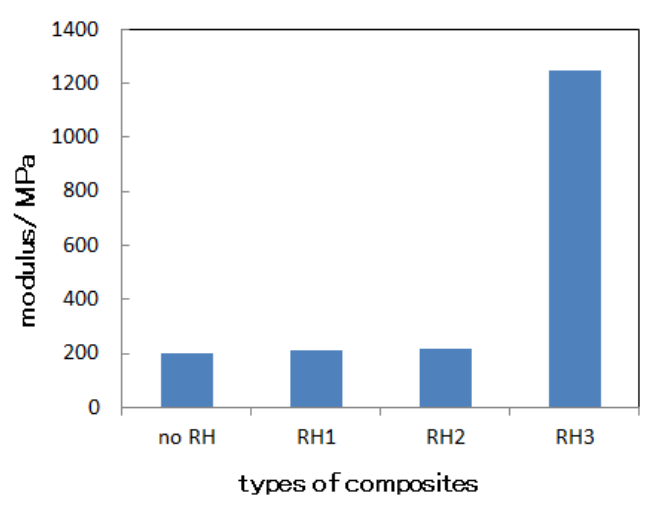

(c) modulus

Fig. 6. Results of the stress at maximum point(a), degree of elongation(b) and elastic modulus(c) for 50 phr of various $\mathrm{RH}$ containing composites under the tensile mode, 
The reason why the composite became brittle by adding the $\mathrm{RH}$ is due to weak interface interaction between the filler and resin, which leads to lower efficiency of mechanical energy transfer from resin to the filler through the interface. While for the modulus, the outstanding increase in RH3 composites was observed. From these observations RH may change the mechanical properties of material to be hard and brittle nature at tensile mode. Fig. 7 shows the results of the mechanical behavior at bending mode.

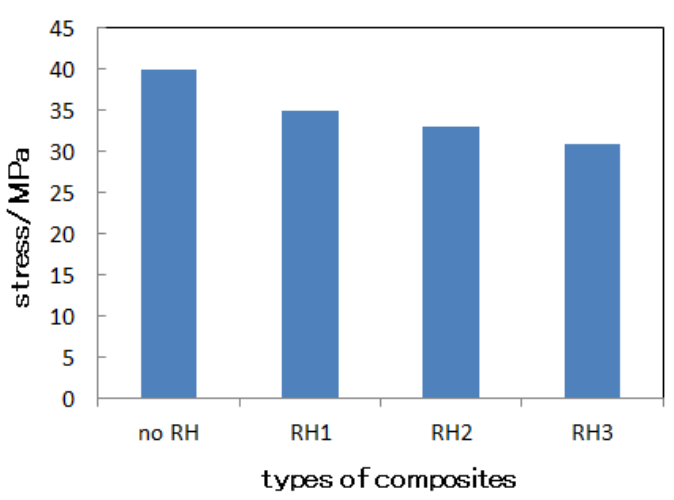

(a)stress at maximum point

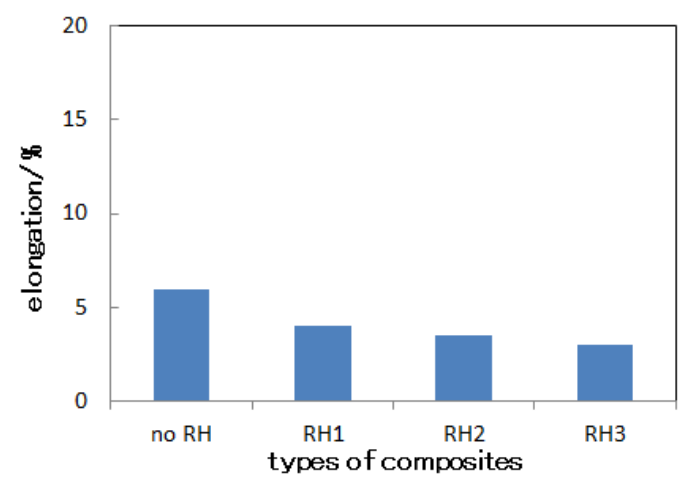

(b) elongation at maximum point

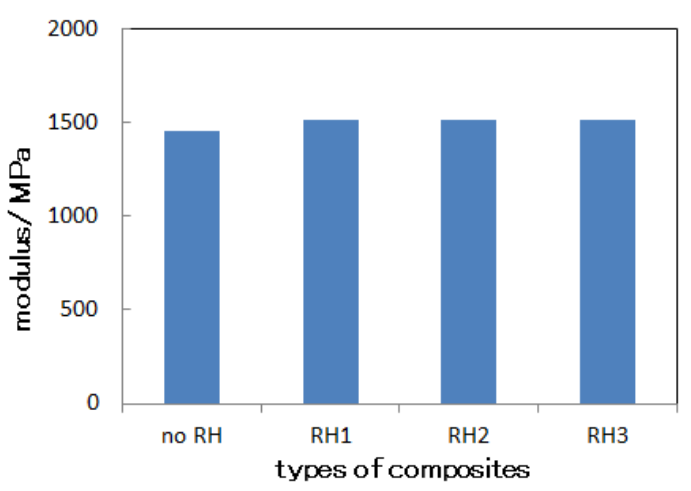

(c) modulus

Fig. 7 Results of the mechanical behavior at bending mode. Explanations of a-c are same as fig. 6 .
As shown in the figure, noticeable changes like a tensile test were not observed. The stress was gradually decreased with decreasing $\mathrm{RH}$ particle size, which was opposite tendency to the tensile mode.

In general, as particle size of the filler become smaller, the tensile strength and modulus of the composites increases. This may be due to the homogeneity on mixture of resin with the fine $\mathrm{RH}$ particles in composites. As well, the surface area between the resin and the RH3 particles is considerably larger than that of RH1 and RH2. Large surface area between the resin and $\mathrm{RH}$ causes the energy transfer more dispersive.

\section{Conclusions}

The composite of resin made from fishing ropes and $\mathrm{RH}$ has been manufactured and the mechanical behavior has been investigated. FTIR and DSC measurements revealed that the fishing ropes used in this study was composed of PP and PE materials. The elastic modulus of the composites in tensile and bending modes increased by adding the RH. While the degree of elongation decreased. Particle size of RH also affects those mechanical properties. The breaking stress and the modulus in the tensile mode substantially increased by using fine particle RH3.

\section{Acknowledgment}

This work was supported by JSPS KAKENHI Grant Number JP16K04799.

\section{References}

(1) T. Ishitani, and K. Ohtsubo: "The Science of Rice (in Japanese)”, Asakura Shoten, Tokyo, 172, 1995

(2) S. Chandrasekhar, K. G. Styanarayama, P. N. Pramada, and P. Raghavan: "Review Processing, properties and applications of reactive silica from rice husk-an overview" J. Mater. Sci., 38, pp. 3159-3168, 2003

(3) M. Shishido, S. Kubo, T. Takahashi and H. Iizuka: Trans. Mat. Res. Soc. Jpn., 31, pp. 993-996, 2006.

(4) M. Shishido, Y. Kurita, M. Ishikawa, and H. Iizuka: Trans. Mat. Res. Soc. Jpn., 32, pp. 1039-1042, 2007

(5) M. Shishido, K. Endo, W. Endo, T. Sato, T. Takahashi and H.Iizuka: "Seawater Resistance of Porous Carbon Materials made from Rice Hull” Trans. Mat. Res. Soc. Jpn., 38, pp. 213-216, 2013

(6) M. Shishido, T. Sato, T. Takahashi, and H. Iizuka: Trans. Mat. Res. Soc. Jpn., 41, pp.63-66, 2016

(7) T. Sato, T. Takumi, S. Takahashi, M. Shishido, and H. Iizuka: "Preparation and Mechanical Properties of Recycled Thermoplastic Composites with Rice Hull Particles”, Trans. Mat. Res. Soc. Jpn., 35, pp. 929-932, 2010 